\title{
Adjunctive Therapy in Oral Cancer
}

\author{
Amit Dhawan
}

\subsection{Introduction}

Oral cancer is the sixth most common cancer in the world, primarily caused by consuming tobacco and its products, alcoholism, nutritional deficiencies, poor oral hygiene, certain strains of human papillomavirus (HPV) and genetic predilection [1, 2]. Considering global incidence of oral cancer, more than $50 \%$ of overall head and neck cancer are occurring in Asian subcontinent with India having maximum numbers of these patients. According to some epidemiological studies in Asian Indian population, approximately 80,000 to $1,20,000$ new patients of oral cancers are being diagnosed yearly, making oral cancer the third most common cancer in India after lung cancer [1]. Oral cavity cancers are primarily treated surgically owing to gross visual disease, easy accessibility, and less chance of obtaining the R1 resection (resection with positive margins). Retrospectively, all T1 and some of $\mathrm{T} 2$ lesions of oral cavity were treated with primary radiotherapy with curative intent, but the results were more promising with upfront surgery followed by some kind of adjuvant treatment depending upon the final histopathology report. Developing countries like India have nonstandardized oral cancer screening protocols due to which most of the oral cancer patients are missed at an early stage and are presented at an advanced stage (stage III-stage IV) with higher T stage (T3-T4) and dissemination of disease in the lymphatics of the neck which further reduces the prognosis for the survivability of the patients. There are other independent predictors on which prognosis of an oral cancer patient depends on like depth of invasion of tumor, involvement of lymph nodes in the neck (lymph node ratio), pattern of invasion of tumor, extranodal extension, and presence of positive margins after surgery. In the recent past, advanced-stage (stage III-stage IV) oral cancer was primarily managed by upfront ablative resection and neck dissection followed by postoperative

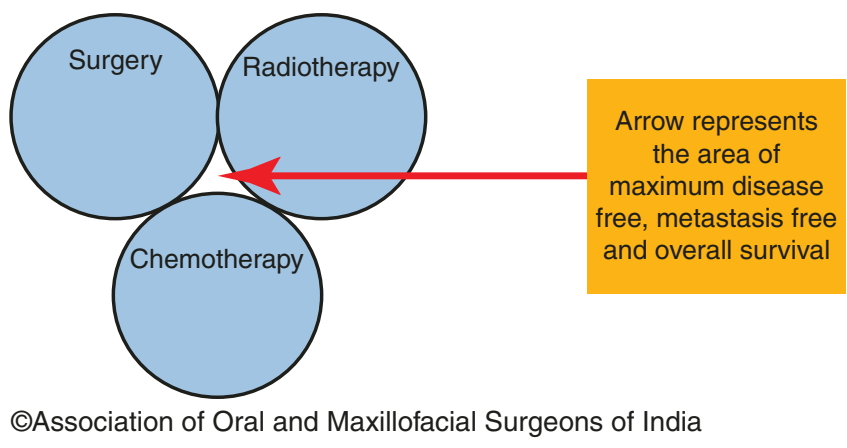

Fig. 84.1 The three major treatment modalities available for the treatment of oral cancer

adjunctive radiotherapy depending upon the other predictors of prognosis. This dual modality approach of followed by radiotherapy has given promising results in advanced-stage cancers, but the overall survival benefit came out to be as low as $40 \%$ [2-5].

In 2004, two large-scale trials were conducted, Radiation Therapy Oncology Group (RTOG) 9501 in America and European organization for Research and Treatment of Cancer (EORTC) 22,931. The results of these trials had provided the new insights for the management of advanced head and neck squamous cell carcinoma. The evidence from these landmark trials confirms the use of chemotherapy to the adjuvant radiation regime in the treatment of advanced-stage squamous cell carcinoma patients $[5,6]$.

The addition of chemotherapy may result in better locoregional control, overall survival, and metastasis-free survival in these patients. The three major pillars of treatment for oral squamous cell carcinoma are surgery, radiotherapy, and chemotherapy. To get the maximum survival advantage from these treatment modalities, all the three treatment modalities have to be used as an adjunct to each other [2, 3] (Fig. 84.1).

\footnotetext{
A. Dhawan $(\varangle)$

Department of Oral and Maxillofacial Surgery, SGRD Dental

Institute, Amritsar, India
} 


\subsection{Basic Principles of Radiotherapy}

When the radiation is used for the purpose of treatment, especially in the patients of oral cancer, it is referred to as radiation therapy or radiotherapy. Radiotherapy is frequently used in as an adjunctive therapy or adjuvant therapy, to improve both local-regional control and overall survival. Radiation therapy is thought to kill tumor cells via damage to their DNA. The amount of radiation is typically measured in the unit gray (Gy), which represents joules of energy per kilogram. The following gives the conversion between grays, radiation absorbed dose (rad), and centigrays: $1 \mathrm{~Gy}=100 \mathrm{rad}=100 \mathrm{cGy}[7,8]$.

External beam radiotherapy refers to the treatment, when a targeted beam of energy in the form of radiation is directed from a machine placed outside the patient to an area to be irradiated located within the patient. However, a radioactive substance may be introduced directly within an existing tumor or tumor-bearing area which is referred to as brachytherapy. Arranging for a patient's radiation treatment involves a number of steps before treatment can commence, of which the first is a dental evaluation and appropriate dental care. After consultation, a patient is simulated, which usually involves obtaining a computed tomographic (CT) scan, while the patient is immobilized with a face mask. Afterward, the radiation oncologist outlines the disease on the scan (contouring). Then a medical physicist and dosimetrist devise a method to deliver the radiation safely to the patient while avoiding radiation to normal anatomical structures. At the time of treatment, the radiation therapists verify that the patient lines up appropriately before treatment can begin. Immobilization of the patient is carried out with a predesigned face mask made up of a thermoplastic material. It is to be noted that accurate positioning of the patient without movement and the ability to recreate the same position of the patient every time (up to 6 weeks) are important factors for overall effective radiation treatment of the patient [7-10].

\subsubsection{Biology of Radiation Therapy}

The current understanding of radiotherapy techniques makes use of radiobiological principles for better treatment planning and outcomes. These principles influence the response of both normal tissues and of tumor clonogens to ionizing radiation, and a basic understanding and application of these principles are vital to improve the narrow therapeutic ratio of tumor versus normal tissue damage.
The 5R' concept of radiobiology is as follows $[9,10]$ :

- Reoxygention: This refers to the process by which surviving hypoxic tumor clonogens become reoxygenated after a period of radiotherapy.

- Repair: This refers to the process by which tumor clonogens damaged by radiation may restore cell integrity to its full potential.

- Redistribution: Distribution of tumor clonogens to a more even cell age distribution after selective cell killing in certain phases of the cell cycle.

- Repopulation: This refers to the process by which tumor clonogens can replenish number of cells following a period of radiation therapy.

- Radiosensitivity: This refers to a dosage of radiation required to make a defined level of cell inactivation after radiation exposure.

\subsubsection{Primary Radiotherapy}

This is also called as radical radiotherapy. The indications of primary radiotherapy only include early-stage oral cancers like carcinoma of lip, ventral surface of tongue, and floor of mouth. According to some reports, primary radiotherapy is indicated for treating $\mathrm{T} 1$ and $\mathrm{T} 2$ cancers with proliferative character. It is believed that the local control and overall survival of the patients are similar in these cases without upfront surgery with an added advantage of preservation of cosmetic, anatomic, and functional integrity of the head and neck region. The literature supports the use of postoperative radiotherapy instead of preoperative radiotherapy even for smallsized tumors as the locoregional recurrence is reported to be slightly more in primary radiotherapy group as compared to postoperative radiotherapy group, but the survival advantage is not statistically significant.

After radiation therapy, the preferred method for assessment of tumor response, in addition to clinical and endoscopic examination, is a posttreatment positron-emission tomographic (PET)/CT scan at 12 weeks. In most patients with a complete response to therapy and negative PET scan results, routine neck dissection is not recommended in the postoperative setting.

\subsubsection{Indications of Adjuvant Radiotherapy}

The treatment for the stage III and stage IV oral cancer is complex. According to current National Comprehensive Cancer 
Network (NCCN) guidelines, single-modality treatment with either surgery or radiotherapy is recommended for approximately $30 \%$ to $40 \%$ of initial stage oral carcinoma patients (stage I and II). Both modalities of treatment have resulted in comparable survival benefit in these patients. On the other hand, combined modality treatment is generally recommended for the approximately $60 \%$ of patients with locally or regionally advanced disease at diagnosis. The treatment in these individuals depends on factors such as site of the disease, stage, pathological findings, and presence of adverse features in the resected specimen. Several studies have classified the oral cancer patients into low risk, medium risk, and high risk [2, 3].

Postsurgical adjuvant radiotherapy is indicated according to the presence of adverse features on final histopathology.

The adverse features refer to extranodal extension, lymphovascular invasion, and perineural invasion.

Patients who are classified as high or intermediate risk of relapse benefit the most from the addition of postoperative radiotherapy.

The indications of adjuvant radiotherapy after primary ablative surgery in oral cancer patients are as follows:

- Stage III and stage IV oral squamous cell carcinoma patients.

- Positive surgical margins: presence of invasive carcinoma at the border.

- Presence of extranodal extension.

- Involvement of more than two levels of lymph nodes (multiple nodal metastasis).

- Perineural invasion.

Often, radiation to the head and neck involves the selection of different doses depending on the amount of disease in that area. Typically, gross disease receives a dose of $70 \mathrm{~Gy}$. Areas at high risk of microscopic disease often receive a dose of between 60 and 66 Gy. Areas at low risk of microscopic disease typically receive a dose of 50-54 Gy. The areas designated at high risk and low risk will depend on the site of occurrence of disease, the stage, and presence of pathological adverse features. The most common fraction size is 2 Gy of radiation per day [10-13].

\subsubsection{Effect of Radiation Treatment Delay}

Postsurgical radiation delay has a profound effect on overall survival of patient. Tumati $\mathrm{V}$ et al. found an association between treatment delays and oncologic outcomes in patients treated with surgery followed by post-op radiotherapy in a total of 277 patients of oral and oropharyngeal carcinoma. On multivariate analysis it was observed that radiation treatment time had a significant impact on locoregional recurrence in these patients. The authors found out that delays in the postoperative radiation therapy $>43$ days were associated with statistically significant locoregional recurrences ( $p=0.02)$ in patients with non-p16-positive cancers. Many investigators have studies in coherence with the above conclusions. An increase in distant metastasis appears to be the mechanism by which prolonged time to treatment initiation leads to worse overall survival [14].

\subsection{Radiotherapy Techniques}

Depending upon the indication and treatment plan, there are multiple techniques with which radiation therapy can be delivered to a patient undergoing treatment for oral cancer. The techniques of radiotherapy should deliver the calculated radiation dose to the patient as per treatment plan without much toxic effects to the normal tissues. Some of the radiation techniques are listed below (Table 84.1) [15-19].

Table 84.1 Radiation techniques

\begin{tabular}{|c|c|}
\hline Radiotherapy technique & Indications \\
\hline $\begin{array}{l}\text { Brachytherapy } \\
\text { (a) Interstitial brachytherapy } \\
\text { (b) Intraluminal } \\
\text { brachytherapy }\end{array}$ & $\begin{array}{l}\text { T1 and T2 tongue and floor of mouth } \\
\text { cancers }\end{array}$ \\
\hline Conventional radiotherapy & For high-risk oral carcinoma patients \\
\hline $\begin{array}{l}\text { Intensity-modulated radiation } \\
\text { therapy (IMRT) }\end{array}$ & $\begin{array}{l}\text { Advanced approach to three- } \\
\text { dimensional treatment planning and } \\
\text { conformal approach. } \\
\text { For dose calculated radiation delivery } \\
\text { to target volume. }\end{array}$ \\
\hline
\end{tabular}

Image-guided radiation therapy (IGRT)
Advances in real-time imaging during radiotherapy

\section{Advantages}

Radiotherapy source placed directly into the tissues to be irradiated. Less chances of systemic toxicities and treatment interruption.

Well established with known pattern of response, local control, and toxicities

Allows for greater sparing of salivary glands, esophagus, optic nerve, spinal cord, and brain stem.

Allows treatment to be delivered in single treatment phase.

Offers the possibility of simultaneously delivering higher radiation to regions of gross disease and lower doses to areas of microscopic disease.

Targeted delivery of radiotherapy which spares the vital structures immediately adjacent to high-dose regions 


\subsubsection{Radiation Dosage and Fractionation Schedule}

Radiotherapy schedule, conventionally, is followed for 5-7 days a week with defined breaks in the treatment for reoxygenation and repair of normal tissue cells of the body for target effectiveness of radiotherapy. According to the literature, the tumor cells repopulate in between the radiotherapy regime especially after fourth week, so unscheduled breaks between the course of radiotherapy are not recommended by the radiation oncologists. That's the reason different fractionation schedules have been advocated by the radiation oncologists for successful completion of the treatment without unnecessary treatment breaks which can have a negative impact on the final outcome of the patients [15-19].

- Conventional fractionation: Every week for 5 days Monday to Friday.

- Hyperfractionation: When more than one fraction of lower radiation dose is delivered to the patient in the same day, it is known as hyperfractionation. The advantage is the treatment can be completed in a shorter time than conventional fractionation, yet the same total dose is delivered with better disease control and without increase in the toxicity.

- Accelerated hyperfractionation: More than one fraction of radiotherapy is given everyday or radiotherapy is given more than 5 days per week, the disadvantage is the increase in the toxicity due to no treatment breaks.

- Continuous hyperfractionated radiotherapy: When the radiation fraction is delivered thrice daily for the whole week, it is known as continuous hyperfractionated radiotherapy. This uninterrupted radiotherapy regime finishes the total dosage of radiation in 12-14 days.

\subsection{Complications of Radiotherapy}

Though the dual-modality treatment (surgery and radiotherapy) has become a norm for the treatment of advanced-stage (stage III-stage IV) oral squamous cell carcinoma, there are some unavoidable toxic effects of radiation therapy which can affect the quality of life (QOL) of the patient. Radiotherapy has proved to be very effective in improving the locoregional control and overall survival of the patient, but there are short-term and long-term sequel of radiotherapy which sometimes limit the effectiveness of the therapy due to breaks in the treatment schedule. The intensity of the complications and toxic effects depends upon the dose of delivered radiation and the site exposed to the radiation therapy. Radiation techniques like intensity-modulated radiotherapy IMRT and image-guided radiotherapy IGRT are helpful in controlling the delivered dosage of radiation to the patient, thereby decreasing the rate of complications and increasing the response of the patient toward the therapy.

Depending upon the response to radiation, the complications have been divided into acute toxic effects or short-term sequel and chronic toxic effects or long-term sequel.

IMRT requires that the radiation oncologist outline the tumor, the areas at risk for microscopic spreading, and the critical normal structures on every CT slice (contouring). Outlining the gross disease (gross tumor volume [GTV]) and areas at risk of microscopic spreading (clinical target volume [CTV]) requires a thorough understanding of the anatomy of the region and is significantly more labor-intensive than prior two-dimensional or conventional radiation therapy techniques.

\subsection{Acute Toxic Effects}

\subsubsection{Xerostomia}

Xerostomia is also referred to as dry mouth. It is one of the most common acute and chronic side effects of radiotherapy. Xerostomia starts from the first week of radiotherapy and is a more common complaint in the first 2 weeks in those patients in whom submandibular salivary glands have been removed as part of the neck dissection. As far as possible, contralateral submandibular salivary gland should be preserved in uninvolved neck when bilateral neck dissection has to be performed. With the traditional radiographic techniques, parotid gland was not spared which resulted in more profound effects of dry mouth leading to halitosis, stasis of thick saliva, dysphagia, and cervical caries. Stasis of thick saliva leads to dysphagia like symptoms and even aggravation of mucositis earlier in the course of therapy.

Intensity-modulated radiotherapy technique and imageguided radiotherapy have revolutionized the concept of delivery of radiotherapy, thereby minimizing the risks of development of unavoidable side effects like xerostomia leading to more acceptable quality of life. A randomized controlled trial by Nutting et al. confirmed the reduction in postradiation xerostomia significantly. They compared intensity-modulated radiotherapy without taking parotid gland in the radiation field and conventional radiation therapy for all patients. About $83 \%$ patients developed grade II or higher xerostomia with conventional radiotherapy as compared to $29 \%$ of patients who were adopted for parotid sparing intensity-modulated radiotherapy [19].

Many investigators have assessed the relationship of radiation dosage to parotid and submandibular gland and severity of xerostomia at weekly intervals. The inference from these studies point toward the damage to plasma membrane of secretory granules of glands. Another observation was that the flow of saliva from submandibular and parotid glands considerably decreases up to less than $20 \%$ of pretreatment 
values by the end of the second week of radiation therapy. The recommendations are to use current radiotherapy techniques like intensity-modulated radiation therapy to spare the submandibular and parotid gland with lowest possible exposure. The treatment of xerostomia converges toward the usage of sialagogues, artificial saliva, and fluid therapy along with reassurance to the patient $[20,21]$.

\subsubsection{Oral Mucositis}

Oral mucositis due to radiotherapy and other systemic therapies in oral cancer treatment represents a major complication causing a wide spectrum of clinical signs and symptoms. Oral mucositis may hamper the quality of life, resulting from debilitating oral pain and bleeding from oral cavity, dysphagia, infections, and inability to chew food and may interfere with the ongoing radiation treatment, ultimately jeopardizing overall patient outcome. According to certain reports, third week of post-radiotherapy is the most crucial time when the mucositis begins to set in and the severity increases in the subsequent weeks. The severity and agony from mucositis is directly proportional to the total dosage of radiation delivered and the volume of irradiated tissue in a specified interval of time. Mild initial erythema to patchy mucositis (characterized by white patches of fibrinous exudate on an erythematous base) to confluent mucositis (where the exudative patches coalesce) are the presenting symptoms of progression of ongoing mucositis in the oral cavity. On the other hand, if there is an evidence of bleeding to touch from the oral ulcers, it is considered as severe to fulminant mucositis.

Confluent mucositis, common toxicity criteria (CTC) grade 3 , is seen in $40-80 \%$ of patients receiving more than 60Gy (or equivalent), more frequently in those receiving concurrent chemotherapy or accelerated radiotherapy. The patients should be advised to keep good oral hygiene with $0.12 \%$ chlorhexidine gluconate along with sialagogues or artificial saliva which will have protective action for the underlying oral mucosa thus reducing the severity of symptoms and relieve the pain. A narcotic analgesic like tramadol is recommended for pain from mucositis. There are some reports which favor elemental zinc or zinc carnosine complex for relieving the symptoms of severe mucositis.

The role of nonalcohol-based mouthwash has been established by many authors for relieving the debilitating effects of mucositis. Topical steroids had been used in past for the treatment of mucositis in severe stages, but their use is constantly discouraged in the current settings. The radioprotectant drug amifostine has been investigated as a means of reducing the incidence of xerostomia and mucositis. The route of administration of amifostine is intravenous everyday prior to initiation of radiotherapy. Patient should be constantly monitored for hypotension and nausea postoperatively.
Moreover, WHO recommends the constant nutrition support to the patients undergoing radiotherapy which will have a positive effect on the recovery from acute mucositis. If oral route is jeopardized, the nasogastric feeding, PEG percutaneous endoscopic gastrostomy, and parenteral nutrition should be considered based on the severity of mucositis. Recovery of severe mucositis may not begin until 2-3 weeks after treatment completion then take up to $4-8$ weeks to regain normal swallowing. The recovery is mostly complete; however, only $<5 \%$ of patients may remain PEG dependent.

Other known acute toxic effects of radiotherapy are erythema of facial and skin of the neck, lethargy, loss of taste, and smell. Erythema of the skin takes about 7-10 days of complete remission after cessation of radiation therapy; however the skin remains photosensitive even after the completion of therapy [20-22].

\subsection{Chronic Toxic Effects}

There are multiple chronic toxic effects of radiotherapy enumerated in the literature by different authors and radiation oncologists (Table 84.2). It has been found out that the chronic effects of radiotherapy are more dependent on dosage than the acute effects of radiation.

\subsubsection{Osteoradionecrosis of Maxilla and Mandible}

Currently, one of the most serious long-term or chronic complications of radiotherapy to the head and neck region is osteoradionecrosis of the jaws. The associated morbidity of this complication and its subsequent treatment will include close observation and/or radical surgical resection followed by reconstruction with free fibula, deep circumflex iliac artery flap (DCIA), or a variety of pedicled and soft tissue free flaps depending upon the severity of necrosis and systemic condition of the patient.

The etiopathogenesis of osteoradionecrosis have been explained on the basis of current theory of Delanian and Lefaix [23]. It states that osteoradionecrosis of the jaws is a radiation-induced fibrosis with histopathological formation of phases which appear similar to those of chronic wounds. There is a histopathological picture of combination of dead or dying osteoblasts without replication of new osteoblasts and excessive proliferation of myofibroblasts. This condition is manifested as loss of trabeculae of existing bone and subsequent decrease in the quality and structure of underlying bone.

Among the risk factors for the development of osteoradionecrosis, total radiation dose delivered to the bone, in a specified period of time, remains the most important and crucial risk factor. The cumulative dose of radiation to the bone will 
Table 84.2 Chronic effects of radiotherapy

\begin{tabular}{l|l} 
Chronic toxicity & Risk factors \\
$\begin{array}{l}\text { Osteoradionecrosis, } \\
\text { mandible, maxilla }\end{array}$ & $\begin{array}{l}\text { Primary bone surgery (marginal mandibulectomy), maxillary } \\
\text { osteoradionecrosis is rare. } \\
\text { Dentulous patients } \\
\text { High radiation dose }>65 \text { Gy to the mandible. } \\
\text { Traumatic extractions. } \\
\text { Women }>\text { men. } \\
\text { Conventional radiotherapy techniques. } \\
\text { Age of the patient. }\end{array}$ \\
\hline Prismus & $\begin{array}{l}\text { Postoperative radiation to the masseter muscle and pterygoid } \\
\text { muscles. }\end{array}$ \\
& $\begin{array}{l}\text { Location of the tumor in the oral cavity. } \\
\text { In case of maxillary tumors, reconstruction with temporalis flap } \\
\text { pose greater risk of developing trismus after radiotherapy }\end{array}$ \\
\hline Hypothyroidism & $\begin{array}{l}\text { Irradiation of thyroid tissue at higher dose. } \\
\text { Female patient }\end{array}$ \\
\hline $\begin{array}{l}\text { Nerve injury (damage to } \\
\text { spinal cord, optic nerve, } \\
\text { vestibulocochlear nerve) }\end{array}$ & $\begin{array}{l}\text { Radiation dosage }>50-54 \text { Gy pose more risk of development of } \\
\text { nerve injuries }\end{array}$ \\
\hline Radiation-induced cancers & $\begin{array}{l}\text { Radiation-induced tumors are mostly sarcomatous and develop } \\
\text { many years after institution of radiotherapy }\end{array}$ \\
\hline
\end{tabular}

\section{Prevention strategy}

IMRT, IGRT, calculating the dose to be delivered to mandible.

Proper dental consultation and rehabilitation before radiotherapy.

Appropriate coverage of remaining bone with free vascularized soft tissue flaps

Early institution of radiotherapy within 4-6 weeks of surgery

Use of microvascular free flaps for reconstruction which provide extra soft and pliable tissue

Dosage to the thyroid gland should be limited.

Use of thyroid shield

IMRT, IGRT, calculating the dose to be delivered

Regular follow-up of the patient produce an environment of hypo-cellularity and hypovascularity leading to the cause and effect relationship in the underlying bone. Maxillary bone is less susceptible to the development of necrosis than mandible as maxilla has a more porous structure of bone with more vascularity as compared to a single terminal inferior alveolar artery in mandible which undergoes fibrosis after high-dose radiation therapy. Moreover, muscles of facial expression and thick mucoperiosteum in maxilla prevent it from getting hypo-vascular in a shorter period of radiation therapy. Facial artery is notoriously unreliable to produce collateral blood supply to the mandible which is insufficient to prevent osteoradionecrosis. On the other hand, in case of post-neck dissection patients, the facial artery is most of the time ligated in the ipsilateral side during the clearance of level IB (submandibular) group of lymph nodes.

According to a comparative study conducted by Kuhnt et al. [22] on 776 patients with head and neck cancer who underwent intensity-modulated radiotherapy as radical radiotherapy for the treatment of head and neck cancer and as postoperative radiotherapy, the highest cumulative incidence of osteoradionecrosis was found to be $12.4 \%$, and relative frequency was $6.6 \%$. The interesting inference of this study is the actual risk for the development of osteoradionecrosis remains there for a lifetime of the patient and may increase by a factor of two in long-term survivors. There remains a highest risk for the development of osteoradionecrosis in patients who had undergone primary bone surgery during tumor resection (hazard ratio: $5.8795 \%$ confidence interval) and in patients with tumor located in the oral cavity than other sub-sites in the head and neck region. This means the dental treatment including extractions and minor surgical procedures should be performed well before in the patients advised for adjuvant radiotherapy in which mandibular resection (marginal mandibulectomy or segmental mandibulectomy) has been advised. Moreover, it is prudent to cover the marginal mandibulectomy defect with the free flap or a soft tissue pedicled flap to maintain vascularity so as to prevent postradiation chance of osteoradionecrosis.

Gender, dentition (dentulous vs edentulous), and concurrent chemotherapy have no significant clinically relevant influence on the development of osteoradionecrosis. Males are found to have three times more risk for the development of osteoradionecrosis than women. According to a study conducted by Gabriela et al., $25 \%$ of patients developed refractory osteoradionecrosis which required some kind of resection and subsequent reconstruction when the radiation dose delivered were more than 66Gy at a daily fraction exceeding up to $2.2 \mathrm{~Gy}$ as compared to no evidence of osteoradionecrosis at or below $65 \mathrm{~Gy}$ of radiation dose [24]. On the other hand, as the daily dose of radiation decreases to 1.8 and $1.9 \mathrm{~Gy}$, and a total dose of 69-75.6 Gy, the rate was $19.6 \%$. Daily dose of radiation is thus considered to be a most significant factor in determining likelihood of developing osteoradionecrosis especially in cases of oral cancer. The close proximity of the cancer to the mandible (with in $1 \mathrm{~mm}$ ) receiving high dose of radiation remains the other risk factors for the development of osteoradionecrosis.

Prevention is better than the treatment of osteoradionecrosis. One study confirms the effectiveness of hyperbaric oxygen therapy in a very few number of patients. State-ofthe-art radiotherapy techniques will limit the total absorbed dose to the mandible and potential sites in the oral cavity thus limiting the incidence of osteoradionecrosis [24, 25]. 


\subsection{Principles of Chemotherapy}

The oral cancer is considered as the sixth most common malignancy worldwide accounting up to $1,30,000$ cases every year in a developing country like India. Due to poor screening facilities at primary examination level, lack of awareness among patients, and nonstandardization of public health sector, most of the cases are diagnosed when they have attained stage III and stage IV disease.

On the other hand, chemotherapy regimens for oral cancer treatment are evolving. There are multiple methods by which chemotherapy may be offered to the patients for the management of head and neck cancer to improve the overall outcomes in terms of survivability of patients.

Methods of providing chemotherapy for head and neck cancer patients are as follows:

- Single-modality treatment for palliation.

- As concomitant radiation therapy or concurrent chemoradiation (triple modality).

- As adjuvant therapy following surgical resection (dual modality).

- As definitive treatment for locoregionally advanced carcinoma primarily.

- As induction therapy or neoadjuvant therapy (NACT) before definitive treatment for prognostication of the primarily inoperable tumors.

Single-modality treatments usually have low efficacy in terms of locoregional recurrences. On the other hand, chemotherapy with or without radiotherapy poses the patient toward the risk of systemic toxicities and unwanted immunosuppression which may alter the overall result and outcomes of the patient undergoing cytotoxic therapy in any form [25-29].

\subsubsection{Cytotoxic Chemotherapeutic Agents}

While multiple cytotoxic drugs have been identified for the treatment of oral squamous cell carcinoma in addition to surgery and radiotherapy, some of the chemotherapy agents are listed below (Table 84.3).

\subsubsection{Chemoradiation in Oral Cavity Cancers}

Earlier it was thought that radiation therapy alone is effective for treating small T1 and T2 lesions of lower lip, floor of mouth, tongue, and palate within the oral cavity. After a decade, many centers adopted surgery alone for smaller lesions of oral cavity as primary treatment. Then a dual-modality approach (surgery followed by radiotherapy) was popularized for locally advanced carcinoma (stage III-stage IV AJCC eighth edition) of oral cavity. Hence, the dual-modality approach emerged as a conventional, widely accepted, and equally effective treatment option for the management of advanced (T3-T4) lesions. Conventionally, the combination of chemotherapy and radiotherapy, as primary treatment modality, had been reserved for those patients with unresectable disease or patients who refuse the surgical treatment.

It has been published by the RTOG trial reports that adjuvant radiotherapy improves the outcome of patients which received the surgical treatment for advanced-stage squamous cell carcinoma arising in the head and neck. At the same time, the disease-free survival and 5-year overall survival came out to be generally between 30 and $40 \%$ which is highly nonpromising. These trials also suggested that if the concurrent chemotherapy, using cytotoxic drugs, is added to the conventional postoperative radiotherapy regime, the effects of radiotherapy are intensified. This methodology of adjunctive treatment has significant amount of survival benefit than dual-modality treatment, and the chances of locore-

Table 84.3 Chemotherapy agents used in oral cancer

\begin{tabular}{l|l|}
$\begin{array}{l}\text { Chemotherapeutic drug } \\
\text { Platinum agents: Cisplatin, } \\
\text { carboplatin, oxaliplatin }\end{array}$ & $\begin{array}{l}\text { Mechanism of action } \\
\text { interstrand cross-links between guanine-guanine pairs } \\
\text { of DNA }\end{array}$ \\
$\begin{array}{l}\text { Taxanes: Paclitaxel, } \\
\text { docetaxel }\end{array}$ & $\begin{array}{l}\text { Act by promoting the formation of tubulin dimers, } \\
\text { stabilizing microtubules during cell division, and } \\
\text { thereby leading to arrest of cells in G2/M phase of cell } \\
\text { cycle }\end{array}$ \\
\hline $\begin{array}{l}\text { 5- fluorouracil: An } \\
\text { antimetabolite }\end{array}$ & $\begin{array}{l}\text { Depletion of nucleotides required for DNA synthesis } \\
\text { and repair }\end{array}$ \\
\hline $\begin{array}{l}\text { Methotrexate } \\
\text { Cetuximab: a monoclonal } \\
\text { antibody against human } \\
\text { EGF receptor }\end{array}$ & $\begin{array}{l}\text { Interferes with the use of folate used for DNA } \\
\text { synthesis and repair inhibits dihydrofolate reductase } \\
\text { (DHFR) }\end{array}$ \\
\hline inhibition of function of the receptor
\end{tabular}

Toxicity and response

Most active agent with response rates 20-50\%. Dose rage is $80-120 \mathrm{mg} / \mathrm{m}^{2}$ every $3-4$ weeks.

Renal toxicity, myelosuppression, neurological, and gastrointestinal toxicity (less with carboplatin)

Particularly severe and life-threatening myelosuppression. Neutropenia, mucositis, and fluid retention

Paclitaxel response up to $37 \%$

Docetaxel response $22-45 \%$

Single-agent response is $15 \%$

Mucositis, diarrhea, myelosuppression

Most commonly used schedule is weekly intravenous bolus regime, using a dose of $40-60 \mathrm{mg} / \mathrm{m}^{2}$. The objective response rate in this dose range is $10-30 \%$ Significantly improved overall survival in head and neck squamous cell carcinoma patients

Consistent severe acneiform skin rash 
gional recurrences are likely to be less especially in high-risk patients postoperatively. In the last 10 years of research in the field of head and neck carcinoma treatment, the efficacy of triple-modality therapy has been justified in the literature for postoperative high-risk squamous cell carcinoma patients. Like RTOG a similar trial was conducted in Europe, the EORTC trial. This trial also reconfirmed the addition of adjuvant chemotherapy to the radiotherapy regime. The inference of this trial showed better rates of local control, diseasespecific survival, and overall survival and significant decreased incidence of late toxic effects. Both these trials compared the addition of three planned cycles of concomitant cisplatin at $100 \mathrm{mg} / \mathrm{m}^{2}$ every 3 weeks to radiotherapy (60-66 Gy, over 6-6.5 weeks, standard fractionation) with the same radiotherapy alone in patients with adverse feature of head and neck carcinoma. Therefore the concomitant therapy with cisplatin at $100 \mathrm{mg} / \mathrm{m}^{2}$ every 3 weeks with adjuvant radiotherapy on day 1 , day 22 , and day 43 is the regimen of choice. The only limitations of these two trials were that these trials were not oral cancer specific. There are very few studies in the literature who have evaluated the concept of triple-modality therapy yet being site specific to oral cavity. Choi et al. retrospectively reviewed 861 patients with oral carcinoma treated in Korea from 1984 to 1996. Almost 63\% of patients had stage III and stage IV cancer, and $36 \%$ had T3-T4 lesions. The authors concluded that triple-modality treatment (upfront surgery followed by postoperative chemoradiation) yielded a greater treatment response rate than dual-modality treatment [29]. On the other hand, Zhang et al. conducted a trial in oral cancer patients who received surgery followed by radiotherapy as a primary treatment modality. The trial found out that there is a 3.6 times more incidence to develop distant metastasis in surgery and radiation group as compared to surgery followed by chemoradiation group [3].

\section{Box 84.1 Questions to be answered}

Now the question arises.

1. Whether the addition of chemotherapy to the radiotherapy regime will improve the survival outcomes?

2. If the survival benefit is observed, does it have any effect on progression-free survival?

3. Is there any of incidence of chronic toxicities if the chemo radiation is used?

4. Does the technique of administering radiation have any profound effect in the outcome?

5. Whether the patients with adverse features (like: extranodal extension, lymphovascular invasion, and perineural spread) have improved outcomes with chemoradiation?
Explanation for Q1\&2: Results of the recent study conducted by Stenson et al. revealed that patients who underwent chemoradiation as primary therapy for advanced-stage oral squamous cell carcinoma had a greater rate of metastasisfree and overall survival benefit with $66.9 \%$ disease-free or progression-free survival [30]. One of the recent singleinstitutional study conducted by Dhawan et al. on 128 patients of advanced-stage high-risk oral carcinoma shows an overall survival benefit of $10 \%$ in patients receiving chemoradiation as compared to patients receiving only radiation therapy after primary ablative surgery. This study was conducted in a subset of Asian Indian population to evaluate the benefits of multimodal therapy in the management of stage III/IV oral carcinoma [2]. Zhang et al. in their study showed a survival benefit of $11 \%$ in patients receiving chemoradiation as compared to radiation alone [3].

Explanation for Q-3: But the survival benefit doesn't come without a price to the patient. Along with improved survival comes the greater incidence of acute and chronic toxicities due to cytotoxic chemotherapeutic agents. The chances of recurrence always remain high even with the addition of chemotherapy due to the advanced stage and higher volume of the disease being treated. The recurrence potential of the lesion depends on the invasive pattern of tumor invasion at the first place and the presence of cancer stem cells in a given area. According to Pericot et al., high incidence of recurrence is associated with size of the primary tumor (T stage), and the difficulty in obtaining margins free of disease, after surgical resection. So obtaining a positive margin or a close margin (between 1 to less than $5 \mathrm{~mm}$ ) after resection becomes an indication for administration of concurrent chemoradiation therapy. On the other hand, the development of myelosuppression, neutropenia, mucositis, and xerostomia is inevitable with triple-modality treatment [31].

Explanation for Q-4: The type of radiation (IMRT and routine radiotherapy) also affects the survival outcomes when used concurrently with chemotherapy as IMRT is more effective along with chemotherapy than with conventional radiotherapy.

Explanation for Q-5: Neck node involvement is also considered as an independent predictor of survival in oral cavity cancer. According to the literature, patients with nonpalpable disease in the neck or clinically N0 necks are having greater overall survival rate as compared to subsequent $\mathrm{N}$ stages. A recent study by Franceschi et al. found that among patients with involved neck nodes, survival rate was significantly lower when more than two levels of lymph nodes were involved, or when extranodal extension was observed. The adverse features which are found after final histopathological examination of primary as well as neck dissection specimen are currently addressed as extranodal extension, perineural invasion, and lymphovascular invasion. These 
patients are particularly at higher risk of disease progression and have higher death rates [32]. According to Zhang et al. [3], patients with extranodal extension and perineural invasion had improved disease-specific and metastasis-free survival in the surgery followed by chemoradiation group $(p<0.05)$. In a retrospective study conducted in 2016, authors found out that overall survival was significantly influenced by the type of modality and regional spread of disease. Chemoradiation after primary ablative surgery group had improved overall, disease-specific, disease-free, and metastasis-free survival compared to surgery followed by radiotherapy group even in patients with extranodal extensions, perineural spread, and lymphovascular invasion.

According to RTOG and EORTC landmark trials, there are some absolute indications for postoperative chemoradiation. These studies provided the base for the risk-adapted strategies in postoperative adjuvant therapy and established extranodal extension and positive surgical margin as the absolute indications for adding concomitant systemic chemotherapy to adjuvant radiation. However, proper patient assessment preoperatively is mandatory keeping in view the systemic toxicity due to chemoradiation.

\section{Absolute Indications of Adjuvant Chemotherapy in Oral Cavity Cancers}

According to RTOG trial, the definition of high-risk patients is as follows:

- Presence of tumor at the surgical resection margins-positive surgical margin.

- Extranodal extension.

- Clinical or radiographic involvement of level IV and level V lymph nodes.

- Stage of pT3 or pT4 with any N except T3N0 of the larynx.

- N2-N3 neck.

- Perineural invasion.

- Vascular embolism.

\subsubsection{Induction Chemotherapy Before Surgery}

Since 1982, during the days of early clinical trials on chemotherapeutic agents and their clinical advantage in head and neck squamous cell carcinoma, induction chemotherapy was thought to have potential benefits for treating head and neck cancers. Unfortunately, the higher response rates of these chemotherapeutic results have failed to result in statistically significant survival benefit. Induction chemotherapy before surgery is generally instituted in primarily unresectable oral cavity cancers at some centers. The efficacy of induction chemotherapy has yet to be proven in oral cancer treatment with regard to locoregional control and/or overall survival of these patients. The efficacy of induction chemotherapy to downstage oral cavity cancers was observed by a study conducted by Grau et al. in a prospective way with unresectable stage III-stage IV oral cavity cancers. They found out that disease-free survival at 5 years was $26 \%$ for patients undergoing resection and $22 \%$ for patients who received chemoradiation. Improved outcome is always at an expense of increased toxicity. There are other studies with comparable results which were carried out exclusively in oral cancer patients. Currently, induction chemotherapy cannot be recommended in resectable oral cancer patients for improvement in the survival [33].

\subsubsection{Palliative Chemotherapy in Patients with Oral Cancer}

Patients with recurrent and metastatic squamous cell carcinoma of head and neck are considered incurable with surgery or radiation. In these patients mainstay of treatment remains the systemic chemotherapy with cytotoxic chemotherapeutic drugs. Chemotherapeutic agents can be used either in combination regimen or as single agent in the management of metastatic oral cavity carcinomas. Radiation therapy with concurrent cetuximab, a monoclonal antibody to epidermal growth factor receptor (EGFR), has been shown to significantly improve overall survival rates compared with radiation therapy alone in the palliative setting. A direct randomized comparison of cetuximab versus platinum-based regimens is underway at many centers in the world. At present, platinum-based regimens (cisplatin, carboplatin) are preferred over other drugs, as long as patients can tolerate the regimen with acceptable complications [34-36].

\subsection{Conclusions}

Adjunctive therapies are included in the treatment regimen of oral squamous cell carcinoma to improve the disease-free, metastasis-free, and overall survival of the patients. Adjuvant radiotherapy is indicated in high-risk advanced-stage (stage III-stage IV) oral squamous cell carcinoma patients as dual modality. The complications and side effects of radiotherapy can be reduced by adopting advanced radiotherapy techniques like intensity-modulated radiotherapy, image-guided radiotherapy, and stereotactic radiotherapy. Concurrent chemoradiation therapy is adopted as primary treatment in patients with locally advanced oral cavity cancer whose disease is not amenable for surgical resection. Adjuvant chemo- 
radiation is indicated in patients having postresection positive margins and with extranodal extensions or extracapsular invasion as a multimodality treatment. Consider cetuximab synchronously with radiotherapy in patients unsuitable for platinum-based chemoradiation.

\section{References}

1. Agarwal AK, Sethi A, Sareen D, Dhingra S. Oral and oropharyngeal squamous cell carcinoma in our population: the clinicpathological and morphological description of 153 cases. Int $\mathbf{J}$ Morphol. 2011;29(3):686-93.

2. Dhawan A, Duggal P, Bhullar RS, Kaur T, Sandhu A, Kaur K. Efficacy of multimodal therapy in the survival outcomes of advanced-stage (stage III-stage IV) oral carcinoma patients: an institutional experience in Asian Indian population. J Maxillofac Oral Surg. 2018;17(1):89-94.

3. Zhang H, Dziegielewski PT, Biron VL, et al. Survival outcomes of patients with advanced oral cavity squamous cell carcinoma treated with multimodal therapy: a multi-institutional analysis. J Otolaryngol Head Neck Surg. 2013;42:30.

4. Cooper JS, Pajak TF, Forastiere A, et al. Precisely defining highrisk operable head and neck tumors based on RTOG \#85-03 and \#88-24:targets for postoperative radiochemotherapy? Head Neck. 1998;20:588-94.

5. Cooper JS, Pajak TF, Forastiere A, et al. Postoperative concurrent radiotherapy and chemotherapy for high risk squamous cell carcinoma of the head and neck. N Engl J Med. 2004;350:19.

6. Bernier J, Cooper JS, Pajak TF, et al. Defining risk levels in locally advanced head and neck cancers: a comparative analysis of concurrent postoperative radiation plus chemotherapy trials of the EORTC (\#22931) and RTOG (\#9501). Head Neck. 2005;27:843-50.

7. Liauw SL, Mancuso AA, Amdur RJ, Morris CG, Villaret DB, Werning JW, et al. Postradiotherapy neck dissection for lymph node-positive head and neck cancer: the use of computed tomography to manage the neck. J Clin Oncol. 2006;24(9):1421-7.

8. Fletcher GH. The role of irradiation in the management of squamous-cell carcinomas of the mouth and throat. Head Neck Surg. 1979;1:441-57.

9. Delclos L, Lindberg RD, Fletcher GH. Squamous cell carcinoma of the oral tongue and floor of mouth. Evaluation of interstitial radium therapy. AJR Am J Roentgenol. 1976;126:223-8.

10. NCCN Clinical Practice Guidelines in Oncology (NCCN Guidelines) Head and Neck Cancers. Version I. 2015. Available at: NCCN.org.

11. Chen PY, Chen HH, Hsiao JR, Yang MW, Hsueh WT, et al. Intensity-modulated radiotherapy improves outcomes in postoperative patients with squamous cell carcinoma of the oral cavity. Oral Oncol. 2012;48:747-52.

12. George JR, Yom SS, Wang SJ. Improved outcomes in adjuvant radiotherapy for oral cavity carcinoma at an academic center: a matched-pair analysis. Laryngoscope. 2014;124:1603-8.

13. Kreppel M, Eich HT, Bruggenolte C, Dreiseidler T, Rothamel D, et al. Preoperative vs. post- operative radiochemotherapy in patients with N2 squamous cell carcinoma of the oral cavity. Oral Oncol. 2012;48:1019-24.

14. Tumati V, Hoang L, Sumer BD, Truelson JM, Myers LL, Khan S, Hughes RS, Nedzi L, Sher DJ. Association between treatment delays and oncologic outcome in patients treated with surgery and radiotherapy for head and neck cancer. Head Neck. 2019 Feb;41(2):315-321.

15. Amit M, Yen TC, Liao CT, Chaturvedi P, Agarwal JP, et al. Improvement in survival of patients with oral cavity squamous cell carcinoma: an international collaborative study. Cancer. 2013;119:4242-8.

16. Hartley A, Cheah N. Postoperative radiotherapy in head and neck cancer. Stell and Maran's textbook of head and neck surgery and oncology, 5th edn, 2012.

17. Rowell N. Principles of radiotherapy. Stell and Maran's textbook of head and neck surgery and oncology, 5th edn, 2012.

18. Sher DJ, Thotakura V, Balboni TA, Norris CM Jr, Haddad RI, et al. Treatment of oral cavity squamous cell carcinoma with adjuvant or definitive intensity-modulated radiation therapy. Int J Radiat Oncol Biol Phys. 2011;81:e215-22.

19. Nutting CM, Morden JP, Harrington KJ, et al. Parotid-sparing intensity modulated versus conventional radiotherapy in head and neck cancer (PARSPORT): a phase 3 multi-centre randomised controlled trial. Lancet Oncol. 2011;12:127-36.

20. Overgaard J, Mohanti BK, Begum N, Ali R, Agarwal JP, et al. Five versus six fractions of radiotherapy per week for squamous-cell carcinoma of the head and neck (IAEA-ACC study): a randomised, multicentre trial. Lancet Oncol. 2010;11:553-60.

21. Beitler JJ, Zhang Q, Fu KK, Trotti A, Spencer SA, et al. Final results of local-regional control and late toxicity of RTOG 9003: a randomized trial of altered fractionation radiation for locally advanced head and neck cancer. Int J Radiat Oncol Biol Phys. 2014;89:13-20.

22. Kuhnt T, Stang A, Wienke A, Vordermark D, Schweyen R, Hey J. Potential risk factors for jaw osteoradionecrosis after radiotherapy for head and neck cancer. Radiat Oncol. 2016;11:101.

23. Delanian S, Lefaix JL. Current management for late normal tissue injury: radiation-induced fibrosis and necrosis. Semin Radiat Oncol. 2007 Apr;17(2):99-107.

24. Studer G, Bredell M, Studer S, et al. Risk profile for osteoradionecrosis of the mandible in IMRT era. Strahlenther Onkol. 2016;192:32.

25. Moon SH, Jung YS, Ryu JS, Choi SW, Park JY, et al. Outcomes of postoperative simultaneous modulated accelerated radiotherapy for head-and-neck squamous cell carcinoma. Int J Radiat Oncol Biol Phys. 2011;81:140-9.

26. Posner MR, Hershock DM, Blajman CR, Mickiewicz E, Winquist $\mathrm{E}$, et al. Cisplatin and fluorouracil alone or with docetaxel in head and neck cancer. N Engl J Med. 2007;357:1705-15.

27. Bernier J, Domenge C, Ozsahin M, Matuszewska K, Lefebvre JL, et al. Postoperative irradiation with or without concomitant chemotherapy for locally advanced head and neck cancer. N Engl J Med. 2004;350:1945-1952.

28. Murthy V, Agarwal JP, Laskar SG, Gupta T, Budrukkar A, et al. Analysis of prognostic factors in 1180 patients with oral cavity primary cancer treated with definitive or adjuvant radio- therapy. J Cancer Res Ther. 2010;6:282-9.

29. Choi KK, Kim MJ, Yun PY, et al. Independent prognostic factors of 861 cases of oral squamous cell carcinoma in Korean adults. Oral Oncol. 2006;42:208-17.

30. Stenson KM, Kunnavakkam R, Cohen EEW, et al. Chemoradiation for patients with advanced oral cavity cancer. Laryngoscope. 2010;120:93-9.

31. Pericot J, Escriba JM, Valdes A, et al. Survival evaluation of treatment modality in squamous cell carcinoma of the oral cavity and oropharynx. J Cranio Maxillofac Surg. 2000;28:49-55.

32. Franceschi D, Gupta R, Spiro RH, et al. Improved survival in the treatment of squamous carcinoma of the oral tongue. Am J Surg. 1993;166:360-5. 
33. Grau JJ, Estapé J, Blanch JL, Vilalta A, Castro V, Biete A, Daniels $M$. Neoadjuvant or adjuvant chemotherapy in the multidisciplinary oral cancer stage III and stage IV. Eur J Cancer B Oral Oncol. 1996;32(4):238-41. issn: 0964-1955

34. Bachaud JM, Cohen-Jonathan E, Alzieu C, David JM, Serrano E, Daly-Schveitzer N. Combined postoperative radiotherapy and weekly cisplatin infusion for locally advanced head and neck carcinoma: nal report of a randomized trial. Int J Radiat Oncol Biol Phys. 1996;36(5):999-1004.
35. Bonner JA, Harari PM, Giralt J, Azarnia N, Shin DM, Cohen RB, et al. Radiotherapy plus cetuximab for squamous-cell carcinoma of the head and neck. N Engl J Med. 2006;354(6):567-78.

36. Denis F, Garaud P, Bardet E, Alfonsi M, Sire C, Germain T, et al. Final results of the 94-01 French head and neck oncology and radiotherapy group randomized trial comparing radiotherapy alone with concomitant radiochemotherapy in advanced-stage oropharynx carcinoma. J Clin Oncol. 2004;22:69-76.

Open Access This chapter is licensed under the terms of the Creative Commons Attribution 4.0 International License (http://creativecommons. org/licenses/by/4.0/), which permits use, sharing, adaptation, distribution and reproduction in any medium or format, as long as you give appropriate credit to the original author(s) and the source, provide a link to the Creative Commons license and indicate if changes were made.

The images or other third party material in this chapter are included in the chapter's Creative Commons license, unless indicated otherwise in a credit line to the material. If material is not included in the chapter's Creative Commons license and your intended use is not permitted by statutory regulation or exceeds the permitted use, you will need to obtain permission directly from the copyright holder. 